\title{
Generating Functions for the Affine Symplectic Group
}

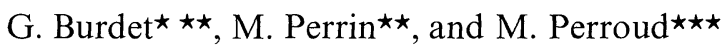 \\ Centre de Recherches Mathématiques, Université de Montréal, Montréal H3C3J7, Canada
}

\begin{abstract}
The generating function notion is used to give a representation of the inhomogeneous symplectic group as group of affine canonical transformations. Then the classical action for linear mechanical systems, the Hamiltonians of which belong to the algebra $h \operatorname{sp}(2 n, \mathbb{R})$, is deduced; it is explicitely constructed for all the Hamiltonians belonging to some particular subalgebras of $h \operatorname{sp}(2 n, \mathbb{R})$. The metaplectic representation of $W \operatorname{Sp}(2 n, \mathbb{R})$ on $L^{2}\left(\mathbb{R}^{n}\right)$ and the solutions of the Schrödinger equation for linear systems are also obtained in terms of generating functions. The Maslov index is explicitly constructed for the quantum corresponding sets of Hamiltonians considered in the classical case.
\end{abstract}

\section{Introduction}

It is well known that the symplectic group $\operatorname{Sp}(2 n, \mathbb{R})$ is connected with $\mathbb{Z}$ as fundamental group. Hence it possesses a non trivial two-sheeted covering $\mathrm{Sp}_{2}(2 n, \mathbb{R})$ a unitary representation of which (now called the metaplectic representation) has been studied in [1] in quantum mechanical and field theory contexts. An explicit realization in terms of integral operators on $L^{2}\left(\mathbb{R}^{n}\right)$ was given in [2], which makes appear the so-called Maslov index [3].

This representation is contained in a projective unitary representation of a larger group the inhomogeneous symplectic group, which is a true unitary representation of the group $W \operatorname{Sp}(2 n, \mathbb{R})$ obtained by extending $\operatorname{Sp}_{2}(2 n, \mathbb{R})$ by the Weyl group.

A large set of quantum mechanical problems is then solved, namely the one's the evolution operator of which is a one-parameter subgroup of $W \operatorname{Sp}(2 n, \mathbb{R})$ (i.e. linear quantum mechanical systems).

* Département de Mathématiques, Université de Montréal, Montréal H3C 3J7, Canada

$\star \star$ Members of the Centre National de la Recherche Scientifique (France)

Present address: Laboratoire de Physique Mathématiques, Faculté des Sciences Mirande, F-21000

Dijon, France

$\star \star \star$ Recipient of aid from the Ministère de l'Education du Gouvernement du Québec 
Recently, a geometrical method to construct the Maslov index for any evolution operator has been given [4], but explicit expressions of this index have been exhibited for few problems only.

In another connection quantum evolution operators for linear systems are merely related to the action of the corresponding classical systems [5], i.e. systems whose evolution operators are one-parameter subgroups of the affine symplectic group $I \mathrm{Sp}(2 n, \mathbb{R})$ : the group of real inhomogeneous linear canonical transformations acting on the phase space.

But it is known that the notion of action originates in the concept of generating function of a canonical transformation. Actually when restricting to the affine group of canonical transformations, this generating function concept will appear as the natural ingredient for constructing the metaplectic representation of this group. In this paper we want to stress on the central role of generating functions in the quantization procedures at least in what concerns linear systems.

In Section 1 we introduce the affine symplectic group $I \operatorname{Sp}(2 n, \mathbb{R})$ and the group $W \operatorname{Sp}(2 n, \mathbb{R})$ generated by $h \operatorname{sp}(2 n, \mathbb{R})$, the one-element central extension of the Lie algebra $i \operatorname{sp}(2 n, \mathbb{R})$.

In Section 2 the generating functions are defined and used to construct a representation of a dense subset of $I \mathrm{Sp}(2 n, \mathbb{R})$.

In Section 3 the classical action of a linear mechanical system is constructed as a family of generating functions and it is explicitely exhibited for Hamiltonians belonging to some particular subalgebras of $h \mathrm{sp}(2 n, \mathbb{R})$. Section 4 is the last section concerning classical mechanics and deals with two practical uses of the generating functions composition law:

i) Possible changes in the choice of the integration constants in the action, related to invariance properties of the Hamilton-Jacobi equation,

ii) Introduction of conjugacy classes of Hamiltonians and of the corresponding actions: a generalization of gauge transformations.

Next we go on to quantum mechanics in Section 5 by introducing the metaplectic representation of $W \mathrm{Sp}(2 n, \mathbb{R})$ on $L^{2}\left(\mathbb{R}^{n}\right)$ in terms of generating functions. In Section 6 solutions of the Schrödinger equation arise as orbits in $L^{2}\left(\mathbb{R}^{n}\right)$ of the one-parameter subgroups of $W \mathrm{Sp}(2 n, \mathbb{R})$. We specially focus our attention upon their continuity properties, and the Maslov indices are explicitely constructed for the sets of Hamiltonians already studied in Section 3.

Finally Section 7 is devoted to some comments about invariance properties of the Schrödinger equation.

\section{The $I \operatorname{Sp}(2 n, \mathbb{R})$ and $W \operatorname{Sp}(2 n, \mathbb{R})$ Groups}

Let $Z=\mathbb{R}^{n} \times \mathbb{R}^{n}=\left\{z=(q, p) \mid q, p \in \mathbb{R}^{n}\right\}$ be a classical phase space. It is a well known result that the Poisson bracket $\{f, h\}$ of two functions in $C^{\infty}(Z)$ induces, through restriction to linear functions and duality, a symplectic structure $\left\langle z, z^{\prime}\right\rangle=-\left\langle z^{\prime}, z\right\rangle$ on $Z$. The invariance group of this form is the symplectic group of $Z$ denoted by $\operatorname{Sp}(2 n, \mathbb{R})$.

The inhomogeneous symplectic group of $Z$ is defined as the semidirect product $I \mathrm{Sp}(2 n, \mathbb{R})=Z \square \mathrm{Sp}(2 n, \mathbb{R})$ with the group law

$$
(z, L)\left(z^{\prime}, L^{\prime}\right)=\left(z+L z^{\prime}, L L^{\prime}\right) \text {. }
$$


The natural action of $I \operatorname{Sp}(2 n, \mathbb{R})$ on $Z\left(g=(z, L): z^{\prime} \mapsto L z^{\prime}+z\right)$ induces a faithful representation $R_{g}: f \mapsto f \circ g^{-1}$ on $C^{\infty}(Z)$ and it turns out that $I \operatorname{Sp}(2 n, \mathbb{R})$ is a group of canonical transformations. (Recall that a diffeomorphism of $Z$ is canonical if it leaves the Poisson bracket invariant.)

By differentiation of $R_{g}$, a faithful representation of the Lie algebra $i \operatorname{sp}(2 n, \mathbb{R})$ is obtained by vector fields on $C^{\infty}(Z)$. Moreover these latter consists of all Hamiltonian vector fields associated to polynomials in $\mathscr{P}_{2}(Z)=\mathscr{P}^{2}(Z)+\mathscr{P}^{1}(Z)$ $+\mathscr{P}^{0}(Z)$, where $\mathscr{P}^{k}(Z)$ denotes the space of homogeneous polynomials of degree $k$ on $Z$. Recall that a hamiltonian vector field $X_{f}$ associated to $f \in C^{\infty}(Z)$ is defined by $X_{f}: h \mapsto\{f, h\}$ and that the mapping $M: \mathrm{f} \mapsto \mathrm{X}_{f}$ is actually a Lie algebra homomorphism between $C^{\infty}(Z)$, considered as a Lie algebra with Poisson bracket, and the Lie algebra of the vector fields on $C^{\infty}(Z)$, with $\mathscr{P}^{0}(Z)$ as kernel. Thus $M: \mathscr{P}_{2}(Z) \rightarrow i \operatorname{sp}(2 n, \mathbb{R})$ is onto and the Lie algebra $h \operatorname{sp}(2 n, \mathbb{R}) \equiv \mathscr{P}_{2}(Z)$ appears as a non trivial extension of $i \operatorname{sp}(2 n, \mathbb{R})$ by a one dimensional Lie algebra. This Lie algebra admits a semidirect decomposition $h \operatorname{sp}(2 n, \mathbb{R})=h_{n} \square \operatorname{sp}(2 n, \mathbb{R})$ where $h_{n} \equiv \mathscr{P}_{1}(Z)$ denotes the Heisenberg algebra (generated by the coordinate functions $Q_{i}, P_{i}$, and 1).

A standard Lie group associated to the Lie algebra $h_{n}$ is the Weyl group $W_{n}=S^{1} \times Z$, with the group law:

$$
(\xi, z)\left(\xi^{\prime}, z^{\prime}\right)=\left(\xi+\xi^{\prime}-\frac{1}{2}\left\langle z, z^{\prime}\right\rangle, z+z^{\prime}\right)
$$

$(\xi \equiv \xi(\bmod 2 \pi))$.

Corresponding to the Lie algebra $h \operatorname{sp}(2 n, \mathbb{R})$, a group $W \operatorname{Sp}(2 n, \mathbb{R})$ can be defined as the semidirect product $W \mathrm{Sp}(2 n, \mathbb{R})=W_{n} \square \operatorname{Sp}(2 n, \mathbb{R})$ with the group law

$$
(\xi, z, L)\left(\xi^{\prime}, z^{\prime}, L^{\prime}\right)=\left(\xi+\xi^{\prime}-\frac{1}{2}\left\langle z, L z^{\prime}\right\rangle, z+L z^{\prime}, L L^{\prime}\right) \text {. }
$$

\section{Generating Functions for $I \operatorname{Sp}(2 n, \mathbb{R})$}

With respect to the natural basis of $Z$, the group $\operatorname{Sp}(2 n, \mathbb{R})$ can be realized as the set of matrices

$$
L=\left(\begin{array}{l}
L_{1} L_{2} \\
L_{3} L_{4}
\end{array}\right), L_{i} \in M_{n}(\mathbb{R}), \text { satisfying } L J^{t} L=J \quad \text { with } \quad J=\left(\begin{array}{rr}
0 & 1 \\
-1 & 0
\end{array}\right)
$$

In order to introduce the various generating functions of affine canonical transformations, we need a particular decomposition of $\operatorname{Sp}(2 n, \mathbb{R})$. Let us introduce the dense subset (which is a open neighbourhood of the identity):

$$
\mathscr{L}_{e}=\left\{L \in \operatorname{Sp}(2 n, \mathbb{R}) \mid \operatorname{det} L_{1} \neq 0\right\} .
$$

Each element of $\mathscr{L}_{e}$ can be decomposed as

$$
L=L_{A} G_{B} \tilde{L}_{C}=\left(\begin{array}{cc}
1 & 0 \\
A & 1
\end{array}\right)\left(\begin{array}{cc}
-B^{-1} & 0 \\
0 & -{ }^{t} B
\end{array}\right)\left(\begin{array}{cc}
1 & -C \\
0 & 1
\end{array}\right), \quad{ }^{t} A=A, \quad{ }^{t} C=C .
$$

In the corresponding decomposition $\mathscr{L}_{e}=\mathscr{A} \mathscr{B} \mathscr{A}$, we notice that $\mathscr{B}$ is a subgroup isomorphic to $\mathrm{Gl}(n, \mathbb{R})$ and that $\mathscr{A}$ and $\tilde{\mathscr{A}}$ are two Abelian subgroups conjugated 
for instance by the inner automorphism

$$
\tilde{L}_{C}=\mathscr{F} L_{(-C)} \mathscr{F}^{-1}, \quad \text { with } \quad \mathscr{F}=\left(\begin{array}{rr}
0 & -1 \\
1 & 0
\end{array}\right) .
$$

The elements (2.1) can be split as

$$
L=\Xi \cdot H^{-1}=\left(\begin{array}{cc}
1 & 0 \\
A & -{ }^{t} B
\end{array}\right)\left(\begin{array}{cc}
-B & C \\
0 & 1
\end{array}\right)^{-1} \text {. }
$$

Both elements $\Xi$ and $H$ of $\operatorname{Gl}(2 n, \mathbb{R})$ do not belong to $\operatorname{Sp}(2 n, \mathbb{R})$; they act on $Z$ as

$$
\Xi:(q, p) \mapsto\left(q, A q-{ }^{t} B p\right) ; \quad H:(q, p) \mapsto(-B q+C p, p) .
$$

Consequently they can be written under the form

$$
\Xi:(q, p) \mapsto\left(q, \nabla_{q} S(q, p)\right) ; \quad H:(q, p) \mapsto\left(\nabla_{p} S(q, p), p\right),
$$

where $\nabla_{q} S$ and $\nabla_{p} S$ denote the partial gradients of the quadratic function

$$
S(q, p)=\frac{1}{2} q \cdot A q-p \cdot B q+\frac{1}{2} p \cdot C p .
$$

We refer to $S(q, p)$ as the generating function of the transformation $L=\Xi \cdot H^{-1}$. We notice that $\operatorname{det}\left(\nabla_{q p}^{2} S(q, p)\right)=\operatorname{det}(-B) \neq 0$.

More generally $[6,7]$, to any differentiable function $S(q, p)$ satisfying the condition $\operatorname{det}\left(\nabla_{q p}^{2} S(q, p)\right) \neq 0$, a canonical transformation $\Phi=\Xi \circ H^{-1}$ is associated through the mappings (2.4). [Indeed, the differential $\left.d\left(\Xi \circ H^{-1}\right)\right|_{(q, p)}$ is of the form (2.3), that is belongs to $\operatorname{Sp}(2 n, \mathbb{R})$.] In particular when $S$ belongs to

$$
\mathscr{P}_{*}^{2}(Z)+\mathscr{P}^{1}(Z)\left(\mathscr{P}_{*}^{2}(Z)=\left\{S \in \mathscr{P}^{2}(Z) \mid \operatorname{det}\left(\nabla_{q p}^{2} S(q, p)\right) \neq 0\right\}\right)
$$

the corresponding canonical transformation belongs to $I \operatorname{Sp}(2 n, \mathbb{R})$.

This procedure defines a one-to-one mapping

$$
\alpha_{e}: \mathscr{P}_{*}^{2}(Z)+\mathscr{P}^{1}(Z) \rightarrow \mathscr{K}_{e}=Z \times \mathscr{L}_{e}
$$

with inverse denoted by $S^{e}\left[\mathrm{so}, S_{g}^{e} \in \mathscr{P}_{*}^{2}(Z)+\mathscr{P}^{1}(Z)\right]$.

Not any element of $I \operatorname{Sp}(2 n, \mathbb{R})$ can be obtained in this way since $\mathscr{K}_{e}$ is only dense in the group. However, for each $h \in I \mathrm{Sp}(2 n, \mathbb{R})$, another dense subset $\mathscr{K}_{h}=\mathscr{K}_{e} \cdot h$ is obtained for which a one-to-one mapping

$$
\alpha_{h}: \mathscr{P}_{*}^{2}(Z)+\mathscr{P}^{1}(Z) \rightarrow \mathscr{K}_{h}
$$

is defined by

$\alpha_{h}(S)=\alpha_{e}(S) \cdot h$.

These various mappings are linked by

$$
\alpha_{h g}=\mu_{g} \circ \alpha_{h}
$$

where $\mu_{g}: g^{\prime} \mapsto g^{\prime} g$ denotes the right multiplication in $I \operatorname{Sp}(2 n, \mathbb{R})$.

In view of the applications to mechanical systems, it is convenient to choose the particular mapping

$$
\alpha_{\mathscr{F}}=\Xi \circ H^{-1} \circ \mathscr{F}
$$


although the identity is not contained in $\mathscr{K}_{\mathscr{F}}$. The canonical transformation $\alpha_{\mathscr{F}}(S)$ corresponding to the generating function in $\mathscr{P}_{*}^{2}(Z)+\mathscr{P}^{1}(Z)$

$$
S(q, p)=\frac{1}{2} q \cdot A q-p \cdot B q+\frac{1}{2} p \cdot C p+d \cdot q+e \cdot p
$$

is, by (2.8), given by

$$
\alpha_{\mathscr{F}}(S):\left(\begin{array}{l}
q \\
p
\end{array}\right) \mapsto\left(\begin{array}{cc}
B^{-1} C & B^{-1} \\
A B^{-1} C-{ }^{t} B & A B^{-1}
\end{array}\right)\left(\begin{array}{l}
q \\
p
\end{array}\right)+\left(\begin{array}{c}
B^{-1} e \\
A B^{-1} e+d
\end{array}\right) .
$$

Conversely, to each element of $\mathscr{K}_{\mathscr{F}}$ corresponds by the inverse mapping $S^{\mathscr{F}}$ a generating function in $\mathscr{P}_{*}^{2}(Z)+\mathscr{P P}^{1}(Z)$; in particular we notice that

$$
S_{g^{-1}}^{\mathscr{F}}(q, p)=-S_{g}^{\mathscr{F}}(p, q), \quad g \in \mathscr{K}_{\mathscr{F}} .
$$

For any chosen $h \in I S p(2 n, \mathbb{R})$, the $I \mathrm{Sp}(2 n, \mathbb{R})$ group law can be lifted from $\mathscr{K}_{h}$ to $\mathscr{P}_{*}^{2}(Z)+\mathscr{P}^{1}(Z)$, at least for those elements $g, g^{\prime} \in \mathscr{K}_{h}$ satisfying $g g^{\prime} \in \mathscr{K}_{h}$, by setting

$$
S_{g g^{\prime}}^{h}=S_{g}^{h}{ }_{h} S_{g^{\prime}}^{h} .
$$

In the case where $h=\mathscr{F}$, we get for $S_{g g^{\prime}}^{\mathscr{F}}$ :

$$
\begin{array}{ll}
A^{\prime \prime}=A-{ }^{t} B\left(A^{\prime}+C\right)^{-1} B & d^{\prime \prime}=d+{ }^{t} B\left(A^{\prime}+C\right)^{-1}\left(e+d^{\prime}\right) \\
B^{\prime \prime}=B^{\prime}\left(A^{\prime}+C\right)^{-1} B & e^{\prime \prime}=e^{\prime}+B^{\prime}\left(A^{\prime}+C\right)^{-1}\left(e+d^{\prime}\right) . \\
C^{\prime \prime}=C^{\prime}-B^{\prime}\left(A^{\prime}+C\right)^{-1 t} B^{\prime} &
\end{array}
$$

It will turn out that this $*$-operation is closely related to the composition of the kernels of the metaplectic representation of $W \operatorname{Sp}(2 n, \mathbb{R})$.

Finally we notice the following property

$$
S_{g}^{h_{1} h_{2}}=S_{g_{h_{1}}}^{h_{1}} S_{h_{2}-1}^{h}
$$

if $h_{2} \in \mathscr{K}_{h_{1}}$ is such that $g h_{2}^{-1} \in \mathscr{K}_{h_{1}}$. Indeed, owing to (2.7), $S_{g}^{h_{1} h_{2}}=S_{g h_{2}^{-1}}^{h_{1}}$, which leads to $(2.14)$.

Traditionally, the generating functions are presented as functions of a mixture of "new" $(q, p)$ and "old" $\left(q_{0}, p_{0}\right)$ variables $\left(g:\left(q_{0}, p_{0}\right) \rightarrow(q, p)\right)$. This does not appear here; however, at a pure formal level, it is simply a way of looking at formulas (2.4) and (2.6). For example, the transformation (2.10) associated to the generating function (2.9) can be obtained from (2.4) and (2.8) by solving the system of equations

$$
p=\nabla_{q} S\left(q, q_{0}\right), \quad p_{0}=-\nabla_{p} S\left(q, q_{0}\right)
$$

in which $S$ appears as a function of the "new" $q$ and of the "old" $q_{0}$. Moreover, the various generating functions associated to a same canonical transformation and related among themselves by various Legendre transforms are all contained in formula (2.14); for example

$$
\begin{aligned}
S_{g}^{e}\left(q, p_{0}\right) & =\left(S_{g}^{\mathscr{F}} * S_{\mathscr{F}}^{\mathscr{F}}\right)\left(q, p_{0}\right) \\
& =p_{0} \cdot q_{0}\left(q, p_{0}\right)+S_{g}^{\mathscr{F}}\left(q, q_{0}\left(q, p_{0}\right)\right)
\end{aligned}
$$

where $q_{0}\left(q, p_{0}\right)$ is the solution of the equation $p_{0}=-\nabla_{q_{0}} S_{g}^{\mathscr{F}}\left(q, q_{0}\right)$. 


\section{Classical Mechanics of Linear Systems}

Let $H \in \mathscr{P}_{2}(Z)$ be the Hamiltonian of a mechanical system. Then $X_{H} \in i \operatorname{sp}(2 n, \mathbb{R})$ and the associated evolution operator $U_{t}$ is a one-parameter subgroup of $I \operatorname{Sp}(2 n, \mathbb{R})$. Let us set

$$
H(q, p)=\frac{1}{2} p \cdot \delta p-q \cdot \gamma p+\frac{1}{2} q \cdot \beta q+\varrho \cdot p+\tau \cdot q
$$

where ${ }^{t} \delta=\delta,{ }^{t} \beta=\beta$ and $\gamma$ belong to $M_{n}(\mathbb{R}), \varrho, \tau \in \mathbb{R}^{n}$.

The Hamilton equations can be written $\frac{d z(t)}{d t}=M z(t)+v$, where

$$
v=\left(\begin{array}{r}
\varrho \\
-\tau
\end{array}\right) \text { and } M=\left(\begin{array}{ll}
-{ }^{t} \gamma & \delta \\
-\beta & \gamma
\end{array}\right) \in \operatorname{Sp}(2 n, \mathbb{R})
$$

after a suitable identification of $X_{H}$ with the pair $(v, M)$. The solution with initial state $z_{0}$ is given by

$$
z(t)=U_{t} z_{0}=(\exp t M) z_{0}+M^{-1}(\exp t M-1) v
$$

if $\operatorname{det} M \neq 0$. If $\operatorname{det} M=0$ the above expression for $U_{t}$ is still formally valid and depends on the nilpotency degree of $M$.

A less direct method known as the Hamilton-Jacobi one consists of expressing the family $\left\{U_{t}\right\}_{t \in \mathbb{R}}$ under the form $U_{t}=\Xi_{t} \circ H_{t} \circ \tau$ by looking for a family of generating functions $\left\{S_{t}^{h}\right\}_{t \in I}$ (we put $S_{t}^{h} \equiv S_{U_{t}}^{h}$ ), the so-called action. Since $U_{t}$ is the flow of the Hamilton equations, it is easy to verify that the following condition on $S_{t}^{h}$ must be satisfied

$$
\partial_{t} S_{t}^{h}+H \circ \Xi_{t}=f(t)
$$

where $f(t)$ is an arbitrary function. Usually this function is taken equal to zero and the above condition is known as the Hamilton-Jacobi equation. Then the action appears as a complete integral of this equation.

In the case under consideration, $U_{t} \in I \operatorname{Sp}(2 n, \mathbb{R})$, then the generating functions $S_{t}^{h}$ are in $\mathscr{P}_{*}^{2}(Z)+\mathscr{P}^{1}(Z)$ but now with coefficients depending upon the parameter $t$. However to solve the Hamilton-Jacobi equation [with $f(t)=0$ ], we have to add a term $F(t)$. This term has to solve the equation

$$
\partial_{t} F=-\frac{1}{2} d \cdot \delta d+\varrho \cdot d .
$$

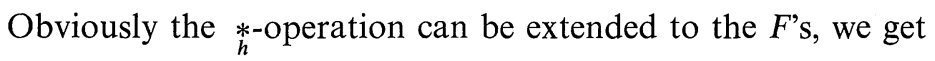

$$
F^{\prime \prime}=F+F^{\prime}-\frac{1}{2}\left(e+d^{\prime}\right) \cdot\left(A^{\prime}+C\right)^{-1}\left(e+d^{\prime}\right) .
$$

The meaning of the last term in (3.4) will become clear in Section 5 .

Since $U_{t}$ is already known from (3.2) and is of the form $U_{t} z_{0}=L(t) z_{0}+W(t)$, with $L(t) \in \mathrm{Sp}(2 n, \mathbb{R})$ and $W(t) \in Z$, the action is immediately obtained by using $(2.10)$ in the case where $h=\mathscr{F}$. It is worth noticing that in general the action is only defined locally, even in the special cases under consideration. Indeed the evolution operator, as a one-parameter subgroup of $I \mathrm{Sp}(2 n, \mathbb{R})$, never is entirely contained in the dense subset $K_{\mathscr{F}}$, in particular $U_{0}=I \notin K_{\mathscr{F}}$. This kind of difficulty does not 
depend on the chosen mapping. For instance if we look for $U_{t}$ under the form $\alpha_{e}\left(S^{\prime}\right)$ instead of $\alpha_{\mathscr{F}}(S)$ as above, the singularity at $t=0$ would be moved at another finite value of $t$ generally.

We give below some examples for which computations can explicitly be done.

\section{Example 1: n-Dimensional Anisotropic Hamiltonians}

By this we mean the Hamiltonians belonging to $h_{n} \square\left(\bigoplus_{1}^{n} \operatorname{sp}(2, \mathbb{R})\right) \subset h \operatorname{sp}(2 n, \mathbb{R})$ which are given by (3.1) where now $\beta, \gamma, \delta$ are diagonal $n \times n$ matrices of elements $\beta_{j}, \gamma_{j}, \delta_{j}$ respectively.

In fact $H=\sum_{j=1}^{n} H_{j}$ with $H_{j} \in h_{1} \square \mathrm{sp}(2, \mathbb{R})$ and it is interesting to note that the quantity $\alpha_{j}^{2}=\gamma_{j}^{2}-\beta_{j} \delta_{j}$ characterizes the one-dimensional subalgebra of $\operatorname{sp}(2, \mathbb{R})$ represented into $H_{j}$.

Then by denoting $\mathscr{V}$ and $\mathscr{W}$ the diagonal $n \times n$ matrices whose elements are $\frac{\operatorname{sh} \alpha_{j} t}{\alpha_{j}}$ and $\operatorname{ch} \alpha_{j} t$ respectively (where $\alpha_{j}=\sqrt{\alpha_{j}^{2}}$ if $\alpha_{j}^{2}>0$ and $\alpha_{j}=i \sqrt{-\alpha_{j}^{2}}$ if $\alpha_{j}^{2}<0$ ), we get $e^{M t}=\left(\begin{array}{cc}\mathscr{W}-\gamma \mathscr{V} & \delta \mathscr{V} \\ -\beta \mathscr{V} & \mathscr{W}+\gamma \mathscr{V}\end{array}\right)$ which leads to the following expression of the action:

$$
\begin{aligned}
S_{t}^{\mathscr{F}}\left(q, q_{0}\right)= & \sum_{j=1}^{n} \frac{\alpha_{j}}{2 \delta_{j} \operatorname{sh} \alpha_{j} t}\left\{\left(\operatorname{ch} \alpha_{j} t+\gamma_{j} \frac{\operatorname{sh} \alpha_{j} t}{\alpha_{j}}\right) q_{j}^{2}-2 q_{j} q_{0 j}+\left(\operatorname{ch} \alpha_{j} t-\gamma_{j} \frac{\operatorname{sh} \alpha_{j} t}{\alpha_{j}}\right) q_{0 j}^{2}\right. \\
& -2\left(\varrho_{j} \frac{\operatorname{sh} \alpha_{j} t}{\alpha_{j}}+\mu_{j} \frac{\operatorname{ch} \alpha_{j} t-1}{\alpha_{j}^{2}}\right) q_{j}+2\left(\varrho_{j} \frac{\operatorname{sh} \alpha_{j} t}{\alpha_{j}}-\mu_{j} \frac{\operatorname{ch} \alpha_{j} t-1}{\alpha_{j}^{2}}\right) q_{0 j} \\
& \left.+\left(\varrho_{j}^{2}-\frac{\mu_{j}^{2}}{\alpha_{j}^{2}}\right) t \frac{\operatorname{sh} \alpha_{j} t}{\alpha_{j}}+2 \frac{\mu_{j}^{2}}{\alpha_{j}^{2}} \frac{\operatorname{ch} \alpha_{j} t-1}{\alpha_{j}^{2}}\right\}
\end{aligned}
$$

where $\mu_{j}=\gamma_{j} \varrho_{j}+\delta_{j} \tau_{j}$. This expression makes clear the fact that the action is never defined for $t=0$. Even more, in the non realistic (from the mechanical point of view) case where any $\delta_{j}=0$, the action is not defined, the corresponding oneparameter subgroup being always outside of the $\mathscr{K}_{\mathscr{F}}$ set, which justifies the choice of the mapping $\mathscr{F}$.

\section{Example 2: The Hamiltonians of the Schrödinger Algebra}

More exactly we are dealing with Hamiltonians which belong to the central extension of the Schrödinger algebra $[8] \widetilde{\mathrm{sch}_{n}} \simeq h_{n} \square(\operatorname{so}(n) \oplus \operatorname{sp}(2, \mathbb{R})) C h \operatorname{sp}(2 n, \mathbb{R})$ and which is given by (3.1) where the matrices $\beta$ and $\delta$ are proportional to the identity matrix and where the matrix $\gamma$ splits into a matrix, always denoted by $\gamma$, proportional to the identity and an antisymmetric one.

Then $e^{M t}=\left(\begin{array}{ll}R & 0 \\ 0 & R\end{array}\right)\left(\operatorname{ch} \alpha t \mathbb{1}_{2 n}+\frac{\operatorname{sh} \alpha t}{\alpha}\left(\begin{array}{cc}-\gamma \mathbb{1}_{n} & \delta \mathbb{1}_{n} \\ -\beta \mathbb{1}_{n} & \gamma \mathbb{1}_{n}\end{array}\right)\right)$ with $\alpha^{2}=\gamma^{2}-\beta \delta\left(\alpha=\sqrt{\alpha^{2}}\right.$ if $\alpha^{2}>0$ and $\alpha=i \sqrt{-\alpha^{2}}$ if $\alpha^{2}<0$ ), and where $R$ is a rotation matrix. The coefficients of the quadratic terms of the action can easily be expressed in terms of the 
parameters of the Hamiltonian, while the expressions of the linear terms coefficients are rather complicated because of the rotations, so we do not give here their explicit expressions and write the action as:

$$
\begin{aligned}
S_{t}^{\mathscr{F}}\left(q, q_{0}\right)= & \frac{\alpha}{2 \delta \operatorname{sh} \alpha t}\left\{\left(\operatorname{ch} \alpha t+\gamma \frac{\operatorname{sh} \alpha t}{\alpha}\right) q^{2}-2 q \cdot R q_{0}+\left(\operatorname{ch} \alpha t-\gamma \frac{\operatorname{sh} \alpha t}{\alpha}\right) q_{0}^{2}\right. \\
& \left.+d(t) \cdot q+e(t) \cdot q_{0}+F(t)\right\} .
\end{aligned}
$$

4.

\subsection{Invariance Properties of the Hamilton-Jacobi Equation}

We just saw that, locally, the evolution operator can be found under the form $U_{t}=\Xi_{t} \circ H_{t}^{-1}$ for example and that the corresponding action is a complete integral of the Hamilton-Jacobi equation $\partial_{t} S_{t}^{e}+H \circ \Xi_{t}=0$. When looking for $U_{t}$ under the form $U_{t}=\Xi_{t}^{\prime} \circ H_{t}^{\prime-1} \circ g$, where $g$ is any transformation of $I \operatorname{Sp}(2 n, \mathbb{R})$, the corresponding action $S_{t}^{g}$ also satisfies the same Hamilton-Jacobi equation. In that sense we can say that $g$ leaves this equation invariant since it maps a complete integral on another one and that, by virtue of (2.14),

$$
S_{t}^{g}=S_{t}^{e} \underset{e}{*} S_{g-1}^{e}
$$

Of course everywhere is local. However, since $\mathscr{K}_{e}$ is an open neighbourhood of the identity, we can say that in the above sense, the Lie algebra $i \operatorname{sp}(2 n, \mathbb{R})$ is an invariance algebra for the Hamilton-Jacobi equation in the class of its complete integrals. A complete integral depending on a set of constants, the can be used to change this set into another one.

\subsection{Conjugacy Classes of Hamiltonians}

In the above discussion, the considered Hamiltonian and consequently the evolution operator remain unchanged under the action of $I \operatorname{Sp}(2 n, \mathbb{R})$, so that we only dealt with a given mechanical system.

Let us now take up an opposite point of view. If $U_{t}$ is an evolution operator associated to a Hamiltonian $H$ and $g$ is any canonical transformation, then the one-parameter group $\hat{U}_{t}=g \circ U_{t} \circ g^{-1}$ can also be considered as an evolution operator associated to the new hamiltonian $\hat{H}=H \circ g^{-1}$ (any canonical transformation permutes the hamiltonian vector fields). When $g$ is in $I \operatorname{Sp}(2 n, \mathbb{R})$ and $H$ is in $h \operatorname{sp}(2 n, \mathbb{R})$, then $H \circ g^{-1}$ is also in $h \operatorname{sp}(2 n, \mathbb{R})$ since $I \operatorname{Sp}(2 n, \mathbb{R})$ acts in this way as an automorphism group. This group action splits the Lie algebra $h \operatorname{sp}(2 n, \mathbb{R})$ into classes of conjugate hamiltonians. The conjugation operation can also be raised upon the set of generating functions by using one of the mappings $S^{h}$ to obtain the new action

$$
\hat{S}_{t}^{h}=S_{g}^{h} \underset{h}{*} S_{t}^{h} \underset{h}{*} S_{g-1}^{h}
$$

which satisfies the Hamilton-Jacobi equation for the transformed Hamiltonian $\hat{H}$. 


\section{The Metaplectic Representation of $W \operatorname{Sp}(2 n, \mathbb{R})$}

The standard way to quantize a classical system, with $\mathbb{R}^{n} \times \mathbb{R}^{n}$ as phase space, consists in realizing the Heisenberg algebra by the following self-adjoint operators on the Hilbert space $\mathscr{H}=L^{2}\left(\mathbb{R}^{n}\right):\left(Q_{k} f\right)(q)=q_{k} f(q),\left(P_{k} f\right)(q)=\frac{\hbar}{i} \partial_{k} f(q)$ and the identity operator.

The corresponding unitary representation of the associated Weyl group is irreducible and is given by

$$
(W(\xi, z) f)(q)=\exp \left\{\frac{i}{\hbar}\left(\xi+q \cdot b-\frac{1}{2} a \cdot b\right)\right\} f(q-a), \quad z=(a, b) .
$$

Owing to its semidirect product structure (1.3), a $W \mathrm{Sp}(2 n, \mathbb{R})$ group representation of the following form can be found

$$
U(\xi, z, L)=W(\xi, z) T(L),
$$

where $T$ is a (projective) unitary representation of $\operatorname{Sp}(2 n, \mathbb{R})$ on $\mathscr{H}$ satisfying

$$
T(L) W(\xi, z) T(L)^{\dagger}=W(\xi, L z) .
$$

We notice that this intertwining condition defines an orbit of representations $W_{L}(\xi, z)=W(\xi, L z)$ of the Weyl group which however are all equivalent. Furthermore, the irreducibility of these representations implies that the representation $T$ satisfying (5.3) is unique up to a phase factor.

Owing to formulas (2.1), (2.2), and (2.8), any element of the dense subset $\mathscr{L}_{\mathscr{F}}$ of $\operatorname{Sp}(2 n, \mathbb{R})$ can be decomposed under the form $L=L_{A} G_{B} \mathscr{F} L_{C}$. Then it is easily verified that the corresponding representative $T(L)=T\left(L_{A}\right) T\left(G_{B}\right) T(\mathscr{F}) T\left(L_{C}\right)$, where

$$
\begin{aligned}
& \left(T\left(L_{A}\right) f\right)(q)=\exp \left\{\frac{i}{2 \hbar} q \cdot A q\right\} f(q) \\
& \left(T\left(G_{B}\right) f\right)(q)=|\operatorname{det} B|^{1 / 2} f(-B q) \\
& (T(\mathscr{F}) f)(q)=(2 \pi \hbar)^{-n / 2} \int_{\mathbb{R}^{n}} d^{n} q_{0} \exp \left\{\frac{i}{\hbar} q \cdot q_{0}\right\} f\left(q_{0}\right),
\end{aligned}
$$

satisfies the Condition (5.3). Hence, up to a yet arbitrary phase factor $\Lambda(L)$, any element $L \in \mathscr{L}_{\mathscr{F}}$ is represented by a unitary integral operator, already considered for instance in $[2,9]$. Next, owing to $(5.2)$, the representative of any element $g=(\xi, z, L)$ with $(z, L) \in \mathscr{K}_{\mathscr{F}}$ is given by a unitary integral operator

$$
(U(g) f)(q)=\int_{\mathbb{R}^{n}} d^{n} q_{0} K_{g}\left(q, q_{0}\right) f\left(q_{0}\right)
$$

with the kernel

$$
\begin{aligned}
K_{g}\left(q, q_{0}\right)= & (2 \pi \hbar)^{-n / 2}|\operatorname{det} B|^{1 / 2} \\
& \cdot \exp \left\{\frac{i \pi}{2} \Lambda(L)+\frac{i}{\hbar}\left[S_{(z, L)}^{\mathscr{F}}\left(q, q_{0}\right)+\xi-\frac{1}{2} B^{-1} e \cdot d\right]\right\}
\end{aligned}
$$


which makes the generating function $S_{(z, L)}^{\mathscr{F}}\left(q, q_{0}\right)$ appear to be associated to $(z, L) \in \mathscr{K}_{\mathscr{F}}$, in which $d=b-A a$ and $e=B a$ [cf. (2.9) and (5.1)].

If $g=(\xi, z, L)$ and $g^{\prime}=\left(\xi^{\prime}, z^{\prime}, L^{\prime}\right)$ are two elements such that $(z, L),\left(z^{\prime}, L^{\prime}\right)$ and the product $\left(z+L z^{\prime}, L L^{\prime}\right)$ are in $\mathscr{K}_{\mathscr{F}}$, it is directly computed from (5.5)-(5.6) that the kernel of $U\left(g^{\prime \prime}\right)=U(g) U\left(g^{\prime}\right)$ is given by

$$
\begin{aligned}
K_{g^{\prime \prime}}\left(q, q_{0}\right)= & (2 \pi \hbar)^{-n / 2}\left|\operatorname{det} B^{\prime \prime}\right|^{1 / 2} \exp \left\{\frac{i \pi}{2} \Lambda\left(L^{\prime \prime}\right)\right. \\
& +\frac{i}{\hbar}\left[\left(S_{(z, L)}^{\mathscr{F}} \frac{*}{\mathscr{F}} S_{\left(z^{\prime}, L^{\prime}\right)}^{\mathscr{F}}\right)\left(q, q_{0}\right)\right. \\
& \left.\left.+\xi+\xi^{\prime}-\frac{1}{2}\left\langle z, L z^{\prime}\right\rangle-\frac{1}{2} B^{\prime \prime}-1 e^{\prime \prime} \cdot d^{\prime \prime}\right]\right\},
\end{aligned}
$$

where the $\frac{*}{\mathscr{F}}$-operation and the quantities $B^{\prime \prime}, e^{\prime \prime}, d^{\prime \prime}$ are defined by (2.12) and (2.13) and

$$
\Lambda\left(L^{\prime \prime}\right)=\Lambda(L)+\Lambda\left(L^{\prime}\right)+\frac{n}{2}-\operatorname{Inert}\left(A^{\prime}+C\right)
$$

$[\operatorname{Inert}(X) \text { denotes the number of negative eigenvalues of } X]^{1}$. The term $-\frac{1}{2} B^{-1} e \cdot d$ in the expression of $K_{g}\left(q, q_{0}\right)$ is a coboundary which will be ignored in what follows. Thus, from the composition law of the kernels, we see that it is possible to associate a generating function to an element $g=(\xi, z, L)$ of $W \operatorname{Sp}(2 n, \mathbb{R})$ simply by setting

$$
S_{g}^{\mathscr{F}}\left(q, q_{0}\right)=S_{(z, L)}^{\mathscr{F}}\left(q, q_{0}\right)+\xi
$$

and to extend the $\mathscr{F}_{\mathscr{F}}$-operation to $\mathscr{P}_{*}^{2}(Z)+\mathscr{P}^{1}(Z)+\mathscr{P}^{0}(Z)$ in order to obtain

$$
S_{g}^{\mathscr{F}} \underset{\mathscr{F}}{*} S_{g^{\prime}}^{\mathscr{F}}=S_{g g^{\prime}}^{\mathscr{F}}
$$

Now, the important problem is to complete the set of all $T(L)$ with $L \in \mathscr{L}_{\mathscr{F}}$ in order to obtain a continuous representation of $\operatorname{Sp}(2 n, \mathbb{R})$. This problem, which has been considered by several authors $[2,3,4]$ has been solved by the introduction of a suitable factor, the so-called Maslov index of $L$. Completed with this index, the representation $T$ is referred as the metaplectic representation of $\operatorname{Sp}(2 n, \mathbb{R})$. It turns out to be a projective representation subduced by a unitary representation of a double covering $\mathrm{Sp}_{2}(2 \mathrm{n}, \mathbb{R})$ of $\operatorname{Sp}(2 n, \mathbb{R})$. The corresponding representation $U$ of $W \mathrm{Sp}(2 n, \mathbb{R})$ is then trivially deduced and also will be called the metaplectic representation of $W \operatorname{Sp}(2 n, \mathbb{R})$. Although an explicit method to compute the Maslov index for any evolution operator in $\operatorname{Sp}(2 n, \mathbb{R})$ has been given in [4], to our knowledge an explicit expression of the Maslov index has been exhibited for two

1 The kernel of the inverse of $U(g)$ is given by [cf. (2.11)]:

$$
K_{g-1}\left(q, q_{0}\right)=(2 \pi \hbar)^{-n / 2}|\operatorname{det} B|^{1 / 2} \exp \left\{-\frac{i \pi}{2}\left(\Lambda(L)+\frac{n}{2}\right)+\frac{i}{\hbar}\left[-S_{(z, L)}^{\mathscr{F}}\left(q_{0}, q\right)+\frac{1}{2}\langle z, L z\rangle-\xi+\frac{1}{2} B^{-1} e \cdot d\right]\right\} .
$$


particular one-parameter subgroups of $\operatorname{Sp}(2 n, \mathbb{R})$ only. In the following section we exhibit some other ones.

\section{Quantum Mechanics of Linear Systems}

For all quantum mechanical systems defined by a Hamiltonian $H$ in $h \operatorname{sp}(2 n, \mathbb{R})$, the evolution operator $U_{t}=\exp \left\{-\frac{i}{\hbar} t H\right\}$ is a one-parameter group of unitary transformations of $\mathscr{H}$ belonging to the metaplectic representation of $W \operatorname{Sp}(2 n, \mathbb{R})$. Thus, by virtue of (5.5)-(5.6), a wave function $\psi_{t}$ with initial value $\phi$ is given by

$$
\begin{aligned}
\psi_{t}(q)= & \left(U_{t} \phi\right)(q)=(2 \pi \hbar)^{-n / 2}\left|\operatorname{det}\left(\frac{\partial^{2} S_{t}^{\mathscr{F}}\left(q, q_{0}\right)}{\partial q_{i} \partial q_{0 j}}\right)\right|^{1 / 2} e^{\frac{i \pi}{2} \Lambda(t)} \\
& \cdot \int_{\mathbb{R}^{n}} d^{n} q_{0} \exp \left\{\frac{i}{\hbar} S_{t}^{\mathscr{F}}\left(q, q_{0}\right)\right\} \phi\left(q_{0}\right) .
\end{aligned}
$$

In all cases the action $S_{t}^{\mathscr{F}}$ is not defined at $t=0$, then the phase $\Lambda(t)$ must be constructed in such a way that the condition $\psi_{0}=\phi$ will be insured for any initial state $\phi$ in $\mathscr{H}$. By using the limit

$$
f(y)=\lim _{\|X\| \rightarrow 0}(2 \pi)^{-n / 2} \frac{e^{-\frac{i \pi}{4}(n-2 \operatorname{Inert}(X))}}{|\operatorname{det} X|^{1 / 2}} \int_{\mathbb{R}^{n}} d^{n} x \exp \left\{(y-x) \cdot X^{-1}(y-x)\right\} f(x),
$$

where $X$ is any symmetric $n \times n$ matrix, it is easy to show that a suitable behaviour of $\psi_{t}$ is obtained if the phase factor behaves like $\frac{n}{2}-\operatorname{Inert}(\delta)$ for small $t$. Then $U_{t}$ is made continuous at $t=0$. But, in general $S_{t}^{\mathscr{F}}$ possesses other singularities for finite values of $t$ which unfortunately are not easily localizable. Therefore we are unable to construct a continuous evolution operator $U_{t}$, for all $t \in \mathbb{R}$, associated to a general $H \in h \mathrm{sp}(2 n, \mathbb{R})$. However in Section 2 the expressions of the classical action have been explicitely given for two particular sets of Hamiltonians, consequently the corresponding Maslov indices can also be explicitly computed by a repeated use of the formula (6.2).

\section{Example 1: The Hamiltonians of the Schrödinger Algebra}

For Hamiltonians belonging to $\widetilde{s \mathrm{ch}_{n}}$ the solution of the corresponding Schrödinger equation is of the general form (6.1) with $S_{t}^{\mathscr{F}}\left(q, q_{0}\right)$ given by (3.6). In this case the only difficulties come from the $\operatorname{sp}(2, \mathbb{R})$ component of the Hamiltonian.

Besides the singularity at $t=0$ above studied for the general case, it also appears singularities for $t=\frac{k \pi}{|\alpha|}$ due to the zeros of the function $\sin |\alpha| t$ when $\alpha^{2}<0$. By inspection it is easy to convince oneself that the wanted continuity properties of the solution can only be obtained with a double covering $\mathrm{Sp}_{2}(2, \mathbb{R})$ of the symplectic group. Hence by a repeated use of one-dimensional version of the 
formula (6.2) we get the phase factor $\Lambda$ which can be written under the form:

$$
\wedge=\operatorname{sig} \delta\left(2-\frac{1}{2} \operatorname{sig} \frac{\operatorname{sh} \alpha t}{\bar{\alpha}}-\operatorname{sig} \frac{\operatorname{sh} \frac{\alpha}{2} t}{\frac{\bar{\alpha}}{2}}\right)
$$

where $\bar{\alpha}$ is the complex conjugate of $\alpha$, in order to cover all cases $\alpha^{2} \gtrless 0$.

The non realistic case $\delta=0$ can now be obtained as a limiting case with the above given $\wedge$. This makes the integral operator disappear (singular kernel). This property, which remains valid in any dimension, will be used in the next section to simplify the restriction of the representation of $W \operatorname{Sp}(2 n, \mathbb{R})$ on the space of solutions to a class of representations of the Schrödinger group. Let us remark that (6.3) is also the right expression of the Maslov index for all unidimensional problems i.e. for Hamiltonians $\in h \mathrm{sp}(2, \mathbb{R})$.

For $n=3$, the so-obtained (spin 0 ) solutions of the Schrödinger equation are exactly the ones coming from the class I projective irreducible unitary representations of $\overline{\mathrm{Sch}}_{3}$ given in [10].

Hence the true quantum mechanical Schrödinger group is

$$
\widetilde{S c h}_{n}=W_{n} \square\left(\operatorname{SO}(n) \otimes \operatorname{Sp}_{2}(2, \mathbb{R})\right) \text {. }
$$

\section{Example 2: n-Dimensional Anisotropic Hamiltonians}

Since this problem consists of a superposition of one-dimensional problems, it is easy to see that $\Lambda=\sum_{j=1}^{n} \Lambda_{j}$ where, for every index $j, \Lambda_{j}$ is given by (6.3) written with the corresponding $\alpha_{j}$,

$$
\Lambda=\sum_{j=1}^{n} \Lambda_{j}=\sum_{j=1}^{n} \operatorname{sig} \delta_{j}\left(2-\frac{1}{2} \operatorname{sig} \frac{\operatorname{sh} \alpha_{j} t}{\bar{\alpha}_{j}}-\operatorname{sig} \frac{\operatorname{sh} \frac{\alpha_{j}}{2} t}{\frac{\bar{\alpha}_{j}}{2}}\right) .
$$

A familiar particular case of such a $n$-dimensional anisotropic Hamiltonian is the case of $n$ harmonic oscillators which is obtained by setting $\delta_{j}=1, \gamma_{j}=\varrho_{j}=\tau_{j}=0$, $\beta_{j}=\omega_{j}^{2},\left|\alpha_{j}\right|=\omega_{j}>0 \forall j \in[1, n]$. Then we get the following expression for the Maslov index:

$$
\Lambda=\sum_{j=1}^{n}\left(2+\frac{1}{2} \operatorname{sig} \sin \omega_{j} t+\operatorname{sig} \sin \frac{\omega_{j} t}{2}\right)
$$

and it is easy to verify that the corresponding solution is exactly the same as the one constructed in [4] by using the pairing techniques.

Finally we want to use the explicit solutions of the one-dimensional problems to discuss the behaviour of these solutions when $\hbar$ becomes negligible compared to the amounts of action involved.

If the $q_{0}$ independent terms, denoted by $\exp \frac{i}{\hbar} S_{t}(q)$, are factorized out of the integral, the limiting procedure (6.2) can be used to evaluate the remaining part of 
the expression. Then it is easy to check that the hybrid function

$$
\tilde{\psi}_{t}(q)=\exp \left\{\frac{i}{\hbar} S_{t}(q)\right\} \times e^{i \frac{\pi}{2}\left(\Lambda(t)+\frac{1}{2} \operatorname{sig}(C)\right)}\left|\frac{B}{C}\right|^{1 / 2} \phi\left(\frac{B q-e}{C}\right)
$$

is exactly the W.K.B. approximation of $\psi_{t}$ at the first order in $\hbar$, i.e.

$$
\text { i) } S_{t}(q)=\frac{1}{2}\left(A-\frac{B^{2}}{C}\right) q^{2}+\left(d+\frac{e B}{C}\right) q+F-\frac{1}{2} B\left(\frac{e}{C}\right)^{2}
$$

is a particular integral of the Hamiltonian-Jacobi equation $\partial_{t} S_{t}+H=0$;

$$
\text { ii) } \begin{aligned}
& X_{t}=\log \left(\left|\frac{B}{C}\right|^{1 / 2} \phi\left(\frac{B q-e}{C}\right)\right) \text { satisfies } \\
& \partial_{t} X_{t}+\left(\varrho-\gamma q+\delta \frac{\partial S_{t}}{\partial q}\right) \frac{\partial X_{t}}{\partial q}=-\frac{1}{2} \delta\left(\frac{\partial^{2} S_{t}}{\partial q^{2}}\right)+\frac{\gamma}{2} .
\end{aligned}
$$

But it should be emphasized that

- the amplitude $X_{t}(q)$ is not a smooth function of $t$.

- the phase $S_{t}(q)$ is not the classical action $S_{t}^{\mathscr{F}}\left(q, q_{0}\right)$.

\section{Invariance Properties of the Schrödinger Equation}

The concept of invariance group for a differential equation in general cannot be defined in a unique way. It depends on the class of the considered solutions and on a particular Ansatz of invariance. During the last five years several papers have been devoted to invariance groups for some Schrödinger equations [11, 12]. In order to replace these ideas in our context, we need to recall that if the space $\mathscr{S}_{H}$ of the solutions of a Schrödinger equation is identified with the Hilbert space of the wave functions $\psi(q, t)=\left(U_{t} \phi\right)(q)$ with $\phi \in \mathscr{H}=L^{2}\left(R^{n}\right)$, there exists an operator $T: \mathscr{H} \rightarrow \mathscr{S}_{H},(T \phi)_{t}=U_{t} \phi$ which is an isometry with the inverse $T^{-1}: \mathscr{S}_{H} \rightarrow \mathscr{H} ; T^{-1} \psi$ $=\psi_{0}$.

The set of all normalized functions $((\psi \mid \psi))=1)$ of $\mathscr{S}_{H}$ is invariant under the whole group $\mathscr{U}\left(\mathscr{S}_{H}\right)$ of the unitary operators; it is therefore the bigger invariance group of the corresponding Schrödinger equation. This is exactly the quantal analogous of the classical property that any canonical transformation on the phase space induces an action on the space of orbits by simply permuting the initial states. More restrictively, any group $G$ for which a unitary representation is defined on $\mathscr{S}_{H}$ is an invariance group. In particular, the group $W \operatorname{Sp}(2 n, \mathbb{R})$ is such an example since the representation $\tilde{U}_{g}=T U_{g} T^{-1}$, where $U_{g}$ denotes the metaplectic representation of this group, is unitary and acts on $\mathscr{S}_{H}$.

The interest offered by an invariance group in general depends on the way it acts on the solutions; some actions are considered as being "natural", for example

$$
\left(\tilde{U}_{g} \psi\right)(q, t)=f_{g}\left(q^{\prime}, t^{\prime}\right) \psi\left(q^{\prime}, t^{\prime}\right) \text {. }
$$

But such a "natural" action can be defined iff for any couple $(g, t)$ there exists a well 
defined couple $\left(g^{\prime}, t^{\prime}\right)$ such that

$$
\left(\tilde{U}_{g} \psi\right)_{t}=\left(T U_{g} T^{-1} \psi\right)_{t}=U_{t} U_{g} \psi_{0}=U_{g^{\prime}} U_{t^{\prime}} \psi_{0}
$$

with

$$
\left(U_{g^{\prime}} \phi\right)(q)=f_{g^{\prime}}\left(q^{\prime}\right) \phi\left(q^{\prime}\right) \text {. }
$$

By simple computation it is seen that the two above conditions can never be realized for the whole group $W \operatorname{Sp}(2 n, \mathbb{R})$ if the evolution law is a subgroup of it.

However, for some $W \operatorname{Sp}(2 n, \mathbb{R})$ subgroups, for example the subgroup $\overline{S c h}_{n}$, it is known [12] that for some special one parameter subgroups $U_{t}$ of $\overline{S \mathrm{ch}}_{n}$, a "natural" action of $\widetilde{S \mathrm{ch}}$ on $\mathscr{S}_{H}$ exists.

Actually the fact that such a possibility exists depends on the value of the parameter $\alpha^{2}$ associated to the Hamiltonian of $U_{t}$ (see Example 2, Section 3). When $\alpha^{2} \leqq 0$, it is always possible to find a $g^{\prime}$ which is represented by a unitary operator with a singular kernel, i.e. of the form (7.3). But for such a choice of $g^{\prime}$ it can be shown that $t^{\prime}$ necessarily is related to $t$ by a "homographic" transformation

$$
f\left(t^{\prime}\right)=\frac{a f(t)+b}{c f(t)+d} \quad \text { where } \quad f(t)=|\alpha| \cot \mathrm{g}|\alpha| t,
$$

where of course $a, b, c, d$ depend on $g$.

On the contrary when $\alpha^{2}>0$ this decomposition of $U_{g} U_{t}$ is not possible, therefore, for instance no "natural" action of $\overline{S c h}_{n}$ exists for an antiharmonic potential. This explains the results given in [12].

Acknowledgements. Two of us, G. Burdet and M. Perrin, are happy to thank the members of the Département de Mathématiques and of the Centre de Recherches Mathématiques of the Université de Montréal for their warm reception. This collaboration was made possible by funds obtained from the Exchanges culturels France-Québec.

\section{References}

1. Shale,D.: Trans. Am. Math. Soc. 103, 149-167 (1962). In another context see also Weil, A. : Acta Math. 111, 143-211 (1963)

2. Leray,J.: Géométrie symplectique et physique mathématique. Aix-en-Provence 1974. Colloq. Intern. Centre Nat. Rech. Sci. Paris 237, 253 (1975)

3. Maslov,V.P., Arnold,V., Bouslaiev,V.: Théorie des perturbations et méthodes asymptotiques. Paris: Dunod 1972

4. Souriau,J.M.: Construction explicite de l'indice de Maslov. In : Proceedings of the $4^{\text {th }}$ International Colloquium on Group Theoretical Methods in Physics, Nijmegen 1975. Lecture notes in physics, Vol. 50, p. 117. Berlin-Heidelberg-New York: Springer 1976

5. Feynman, R.P.: Rev. Mod. Phys. 20, 367-387 (1948)

6. Arnold,V.: Méthodes mathématiques de la mécanique classique. Moscou: Editions Mir 1976

7. Sternberg,S.: Celestial mechanics. II. New York: W. A. Benjamin Inc. 1969

8. Burdet,G., Perrin, M. : J. Math. Phys. 16, 2172-2176 (1975)

9. Moshinsky,M., Quesne, C.: J. Math. Phys. 12, 1772-1780 (1971)

10. Perroud, M.: Helv. Phys. Acta 50, 233-252 (1977)

11. Hagen, C. R.: Phys. Rev. D 5, 377-388 (1972)

12. Niederer, U.: Helv. Phys. Acta. 45, 802-810 (1972); 46, 191-200 (1973)

\section{Communicated by W. Hunziker}

Received January 5, 1977; in revised form November 14, 1977 